\section{KRUKENBERG TUMORS: GASTRIC CANCER METASTASIS}

S Saidi*, S Ghomari. Medical Oncology Department - CHU Tlemcen Laboratoire Toxicomed. Department de Medecine. Universite dTlemcen, Tlemcen, Algeria

\subsection{6/ijgc-2021-ESGO.488}

Introduction/Background* Krukenberg tumors (TK) are defined as bilateral ovarian metastases, they are rare and represent 1 to $2 \%$ of ovarian tumors. They are secondary to digestive tumors and mainly of gastric origin. Often detected at an advanced stage of the disease, the survival prognosis is poor despite chemotherapy, with or without surgical resection.

Methodology A retrospective study on the files of patients treated, in the Medical Oncology department -Tlemcen Hospital from 2010 to 2020, was realized.

The goal of our work is to determine the ovarian metastases frequency in the course of gastric cancers as well as the clinical and therapeutic characteristics of this secondary location.

Result(s)* Six cases have been reported. The mean age was 52 years $[31,66]$. The gastric neoplasia was diagnosed by epigastralgia, deterioration of performance status, vomiting, anorexia and weight loss. The gastric endoscopy exploration revealed antral tumor in five patients and subcardial tumor in one case. The anatomo-pathologic study found a moderately differentiated gastric adenocarcinoma and the signet ring cell carcinoma $(3 / 3)$.

A total gastrectomy with lymph node dissection was performed in three patients, one of whom was after perioperative chemotherapy.

Ovarian metastases were synchronous in four patients and metachronous in two patients after a free interval of 16 months and 30 months respectively. Ovarian metastases were bilateral and were revealed by abdomino-pelvic CT. Only one patient was operated, a total hysterectomy with bilateral oophorectomy was performed. Three patients received palliative chemotherapy. Only supportive care was offered in two patients.

Conclusion* Krukenberg tumors are rare. The diagnosis is facilitated by pelvic ultrasound performed as part of the assessment of the extension of a cancer of digestive or extradigestive location. Advances in chemotherapy could improve the prognosis of these tumors, which remains bleak.

\section{CARBOPLATIN FOLLOWD BY OLAPARIB VERSUS BEVACIZUMAB IN MANTEINANVE THERAPY IN ELDERLY PATIETS WHIT ADVANCED OVARIAN CANCER}

ST Miano*, G Francini, I Martellucci. UOC Oncologia medica, azienda Ospedaliera Universitaria Senese, siena, Italy

\subsection{6/ijgc-2021-ESG0.489}

Introduction/Background* *: The poly(ADP-ribose) polymerase inhibitor olaparib has shown antitumor activity in patients with platinum-sensitive, recurrent, high-grade serous ovarian cancer with or without BRCA1 or BRCA2 mutations. The aim of our study was to assess the efficacy and tolerability of Carboplatin in single agent therapy, followed by olaparib maintenance monotherapy, versus manteinance therapy bevacizumab in elderly patients with platinum-sensitive, recurrent, high-grade serous ovarian cancer according to BRCA status.

Methodology In our retrospective study, old patients (median age 80) with platinum-sensitive, recurrent, high-grade serous ovarian cancer received carboplatin (area under the curve [AUC] $4 \mathrm{mg} / \mathrm{mL}$ per min, according to the Calvert formula, administered intravenously on day 1)followed by olaparib monotherapy (400 mg capsules twice daily, given continuously) or Bevacizumab $15 \mathrm{mg} / \mathrm{kv}$ ev on day 1 every 21 days until progression The primary endpoint was progression-free survival.

Result(s)* Between Feb 17 and July 30, 2020, 17 patients were eligible and were assigned to the two treatment groups (5 to the olaparib group and 12 to the bevacizumab group). BRCA mutation status was known for all patients (either at baseline or determined retrospectively): 5 of 17 had a BRCA mutation Progression-free survival was significantly longer in the olaparib group (median 24.2 months [95\% CI 9.7-15.0]) than in the bevacizumab group (median 9.6 months [95\% CI 9.1-9.7) (HR 0.51 [95\% CI 0.34-0.77]; $p=0.0012$ ), especially in patients with BRCA mutations (HR 0.21 [0.08-0.55]; $\mathrm{p}=0.0015)$. Adverse events more commonly reported in the olaparib group than in the placebo group (by more than $10 \%$ of patients) were nausea $(68 \%$ vs. $35 \%)$, fatigue (49\% vs. $38 \%)$, vomiting ( $32 \%$ vs. $14 \%)$, and anemia ( $17 \%$ vs. $5 \%)$; the majority of adverse events were grade 1 or 2 .

Conclusion* *: Carboplatin in monotherapy followed by olaparib in manteinance therapy significantly improved progression-free survival versus bevacizumab plus carboplatin alone, with the greatest clinical benefit in BRCA-mutated patients elderly patients and had an acceptable and manageable tolerability profile.

\section{INCIDENCE OF OVARIAN METASTASIS FROM NON- GENITAL TRACT PRIMARY TUMOR SITES}

${ }^{1} \mathrm{C}$ Theofanakis*, ${ }^{1} \mathrm{~K}$ Trimmi, ${ }^{2} \mathrm{M}$ Liontos, ${ }^{1} \mathrm{~V}$ Pergialiotis, ${ }^{1} \mathrm{~V}$ Theodoulidis, ${ }^{1} \mathrm{DE}$ Vlachos, ${ }^{1} \mathrm{~N}$ Thomakos, ${ }^{1} \mathrm{~A}$ Rodolakis, ${ }^{1} \mathrm{D}$ Haidopoulos. 'University of Athens, Division of Gynaecological Oncology, 1st Department of Obstetrics and Gynaecology, Alexandra Hospital, Athens, Greece; ${ }^{2}$ University of Athens, Oncology Unit, Department of Clinical Therapeutics, Alexandra Hospital, Athens, Greece., Greece

\subsection{6/ijgc-2021-ESGO.490}

Introduction/Background* Management of a pelvic mass is a common cause of surgery among women. In nearly $20 \%$ of cases, such masses represent primary ovarian malignancies, treated with complete surgical staging. However, in $6-7 \%$ of cases, the ovarian malignancy will present as a metastatic lesion form other sites, known as Krukenberg tumors. This term usually characterizes primary gastric cancer, but Krukenberg tumors can also arise from other primary sites. In the present study we assessed the characteristics of metastatic tumors to the ovaries from non-genital tract primary sites and attempted to determine the route of tumor spread.

Methodology We retrospectively reviewed medical records of patients whose indication for surgery was a pelvic mass from January 2000 to December 2018. The study was conducted after approval of the Institutional Review Board. Reports and medical files were reviewed for age at diagnosis, tumor size, laterality of metastasis and primary tumor site. Only patients with metastatic disease to the ovary were included in the study. Result(s)* A total of 64 cases of metastasis to the ovary were identified. The median age the patients was 58 years old (range: 28 - 81). Primary gastric cancer was identified in 28 (43.9\%) cases; breast cancer 14 (21.9\%) and colon cancer 13 (20.3\%). Pancreatic cancer and urinary bladder each contributed $3(4.7 \%)$ cases, while b-cells lymphoma, primary 